\title{
The doubloon polynomial triangle
}

\author{
Dominique Foata and Guo-Niu Han \\ Dedicated to George Andrews, \\ on the occasion of his seventieth birthday.
}

\begin{abstract}
The doubloon polynomials are generating functions for a class of combinatorial objects called normalized doubloons by the compressed major index. They provide a refinement of the $q$-tangent numbers and also involve two major specializations: the Poupard triangle and the Catalan triangle.
\end{abstract}

\section{Introduction}

The doubloon $\left(^{*}\right)$ polynomials $d_{n, j}(q)(n \geq 1,2 \leq j \leq 2 n)$ introduced in this paper serve to globalize the Poupard triangle [Po89] and the classical Catalan triangle [S107]. They also provide a refinement of the q-tangent numbers, fully studied in our previous paper [FH08]. Finally, as generating polynomials for the doubloon model, they constitute a common combinatorial set-up for the above integer triangles. They may be defined by the following recurrence:

(D1) $d_{0, j}(q)=\delta_{1, j}($ Kronecker symbol);

(D2) $d_{n, j}(q)=0$ for $n \geq 1$ and $j \leq 1$ or $j \geq 2 n+1$;

(D3) $d_{n, 2}(q)=\sum_{j} q^{j-1} d_{n-1, j}(q)$ for $n \geq 1$;

(D4) $d_{n, j}(q)-2 d_{n, j-1}(q)+d_{n, j-2}(q)$

$$
\begin{aligned}
= & -(1-q) \sum_{i=1}^{j-3} q^{n+i+1-j} d_{n-1, i}(q) \\
& -\left(1+q^{n-1}\right) d_{n-1, j-2}(q)+(1-q) \sum_{i=j-1}^{2 n-1} q^{i-j+1} d_{n-1, i}(q)
\end{aligned}
$$

for $n \geq 2$ and $3 \leq j \leq 2 n$.

The polynomials $d_{n, j}(q)(n \geq 1,2 \leq j \leq 2 n)$ are easily evaluated using $(D 1)-(D 4)$ and form the doubloon polynomial triangle, as shown in Fig. 1.1 and $1.1^{\prime}$.

$$
\begin{aligned}
& d_{1,2}(q) \\
& d_{2,2}(q) d_{2,3}(q) d_{2,4}(q) \\
& d_{3,2}(q) d_{3,3}(q) d_{3,4}(q) d_{3,5}(q) d_{3,6}(q) \\
& d_{4,2}(q) d_{4,3}(q) d_{4,4}(q) d_{4,5}(q) d_{4,6}(q) d_{4,7}(q) d_{4,8}(q)
\end{aligned}
$$

Fig. 1.1. The doubloon polynomial triangle

Key words and phrases. Doubloon polynomials, doubloon polynomial triangle, Poupard triangle, Catalan triangle, reduced tangent numbers.

Mathematics Subject Classifications. 05A15, 05A30, 33B10

$(*)$ Although the word "doubloon" originally refers to a Spanish gold coin, it is here used to designate a permutation written as a two-row matrix. 


$$
\begin{aligned}
& d_{1,2}(q)=1 ; \quad d_{2,2}(q)=q ; \quad d_{2,3}(q)=q+1 ; \quad d_{2,4}(q)=1 ; \\
& d_{3,2}(q)=2 q^{3}+2 q^{2} ; \quad d_{3,3}(q)=2 q^{3}+4 q^{2}+2 q ; \quad d_{3,4}(q)=q^{3}+4 q^{2}+4 q+1 ; \\
& d_{3,5}(q)=2 q^{2}+4 q+2 ; \quad d_{3,6}(q)=2 q+2 ; \\
& d_{4,2}(q)=5 q^{6}+12 q^{5}+12 q^{4}+5 q^{3} ; \quad d_{4,3}(q)=5 q^{6}+17 q^{5}+24 q^{4}+17 q^{3}+5 q^{2} ; \\
& d_{4,4}(q)=3 q^{6}+15 q^{5}+29 q^{4}+29 q^{3}+15 q^{2}+3 q ; \\
& d_{4,5}(q)=q^{6}+9 q^{5}+25 q^{4}+34 q^{3}+25 q^{2}+9 q+1 ; \\
& d_{4,6}(q)=3 q^{5}+15 q^{4}+29 q^{3}+29 q^{2}+15 q+3 ; \\
& d_{4,7}(q)=5 q^{4}+17 q^{3}+24 q^{2}+17 q+5 ; \quad d_{4,8}(q)=5 q^{3}+12 q^{2}+12 q+5 .
\end{aligned}
$$

Fig. 1.1'. The first doubloon polynomials

Notice the different symmetries of the coefficients of the polynomials $d_{n, j}(q)$, which will be fully exploited in Section 4 (Corollaries 4.3, 4.7, 4.8). Various specializations are displayed in Fig. 1.2 below, where $C_{n}=$ $\frac{1}{2 n+1}\left(\begin{array}{c}2 n \\ n\end{array}\right)$ stands for the celebrated Catalan number and $t_{n}$ for the reduced tangent number occurring in the Taylor expansion

$$
\begin{aligned}
\sqrt{2} \tan (u / \sqrt{2}) & =\sum_{n \geq 0} \frac{u^{2 n+1}}{(2 n+1) !} t_{n} \\
& =\frac{u}{1 !} 1+\frac{u^{3}}{3 !} 1+\frac{u^{5}}{5 !} 4+\frac{u^{7}}{7 !} 34+\frac{u^{9}}{9 !} 496+\frac{u^{11}}{11 !} 11056+\cdots
\end{aligned}
$$

The symbol $\Sigma$ attached to each vertical arrow has the meaning "make the summation over $j$ " and $d_{n}(q)$ is the polynomial further defined in (1.3).

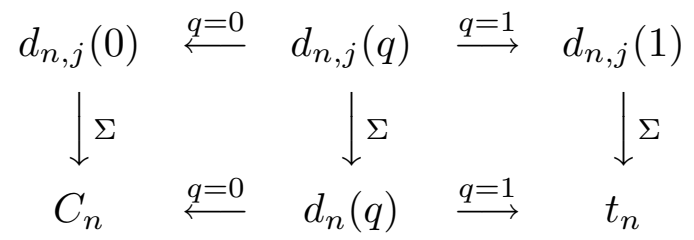

Fig. 1.2. The specializations of $d_{n, j}(q)$

When $q=1$, the $(D 1)-(D 4)$ recurrence becomes

$(P 1) d_{0, j}(1)=\delta_{0, j}$ (Kronecker symbol);

$(P 2) d_{n, j}(1)=0$ for $n \geq 1$ and $j \leq 1$ or $j \geq 2 n+1$;

$(P 3) d_{n, 2}(1)=\sum_{j} d_{n-1, j}(1)$ for $n \geq 1$;

$(P 4) d_{n, j}(1)-2 d_{n, j-1}(1)+d_{n, j-2}(1)=-2 d_{n-1, j-2}(1)$

for $n \geq 2$ and $3 \leq j \leq 2 n$,

which is exactly the recurrence introduced by Christiane Poupard [Po89].

$$
\begin{array}{ccccc} 
& & 1 & & \\
& 1 & 2 & 1 & \\
4 & 8 & 10 & 8 & 4
\end{array}
$$

346894104946834

Fig. 1.3. The Poupard triangle $\left(d_{n, j}(1)\right)$ 
The integers $d_{n, j}(1)$ are easily evaluated using $(P 1)-(P 4)$ and form the Poupard triangle, as shown in Fig. 1.3.

When $q=0$, relation $(D 4)$ becomes:

$$
d_{n, j}(0)-2 d_{n, j-1}(0)+d_{n, j-2}(0)=-d_{n-1, j-2}(0)+d_{n-1, j-1}(0),
$$

which can be rewritten as

$$
d_{n, j}(0)-d_{n, j-1}(0)-d_{n-1, j-1}(0)=d_{n, j-1}(0)-d_{n, j-2}(0)-d_{n-1, j-2}(0),
$$

so that by induction

$$
d_{n, j}(0)-d_{n, j-1}(0)-d_{n-1, j-1}(0)=d_{n, 2}(0)=\left.\sum_{j} q^{j-1} d_{n-1, j}\right|_{q=0}=0
$$

using (D2) and (D3) when $n \geq 2$. Consequently, the integers $d_{n, j}(0)$ satisfy the recurrence relation

$$
d_{n, j}(0)=d_{n, j-1}(0)+d_{n-1, j-1}(0)
$$

for $n \geq 2$ and $3 \leq j \leq 2 n$ with the initial conditions

$$
\begin{gathered}
d_{n, n+1}(0)=1 \quad(n \geq 1) \\
d_{n, j}(0)=0 \quad(n \geq 1 \text { and } j \leq 1 \text { or } j \geq 2 n+1) \\
d_{n, 2}(0)=d_{n, 3}(0)=\cdots=d_{n, n}(0)=0 \quad(n \geq 2) .
\end{gathered}
$$

In view of $(C 1)$ and $(C 2)$ the integers $d_{n, j}(0)(n \geq 1, n+1 \leq j \leq 2 n)$ obey the rules of the classical Catalan triangle that has been studied by many authors (see the sequence A00976 in Sloane [S107] and its abundant bibliography). They form the Catalan triangle displayed in Fig. 1.4.

\author{
1 \\ 011 \\ 00122

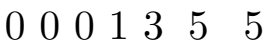 \\ $\begin{array}{llllllllll}0 & 0 & 0 & 0 & 1 & 4 & 9 & 14 & 14\end{array}$ \\ 0000001514284242
}

Fig. 1.4. The Catalan triangle $\left(d_{n, j}(0)\right)$

For each $n \geq 0$ let $A_{n}(t, q)$ be the Carlitz [Ca54, Ca75] $q$-analog of the Eulerian polynomial defined by the identity

$$
\frac{A_{n}(t, q)}{(t ; q)_{n+1}}=\sum_{j \geq 0} t^{j}\left([j+1]_{q}\right)^{n},
$$

where $(t ; q)_{n+1}=(1-t)(1-t q) \cdots\left(1-t q^{n}\right)$ and $[j+1]_{q}=1+q+q^{2}+\cdots+q^{n}$ are the traditional $q$-ascending factorials and $q$-analogs of the positive integers. 
The polynomial $d_{n}(q)$ under study was introduced in [FH08]. It is defined by

$$
d_{n}(q)=\frac{(-1)^{n} q^{\left(\begin{array}{c}
n \\
2
\end{array}\right)} A_{2 n+1}\left(-q^{-n}, q\right)}{(1+q)\left(1+q^{2}\right) \cdots\left(1+q^{n}\right)}
$$

It was shown to be a polynomial of degree $\left(\begin{array}{c}n-1 \\ 2\end{array}\right)$, with positive integral coefficients, having the following two properties:

$$
\begin{aligned}
& d_{n}(1)=t_{n} \\
& d_{n}(0)=C_{n} \quad(n \geq 0)
\end{aligned}
$$

(see the bottom row in the diagram of Fig. 1.2.)

The first values of the polynomials $d_{n}(q)$ are: $d_{0}(q)=d_{1}(q)=1$; $d_{2}(q)=2+2 q ; d_{3}(q)=5+12 q+12 q^{2}+5 q^{3} ; d_{4}(q)=14+56 q+110 q^{2}+$ $136 q^{3}+110 q^{4}+56 q^{5}+14 q^{6}$.

The main purpose of this paper is to prove the following theorem.

Theorem 1.1. Let $\left(d_{n, j}(q)\right)$ be the set of polynomials defined by $(D 1)-$ $(D 4)$ and $d_{n}(q)$ be defined by (1.3). Then, the following identity holds

$$
\sum_{j} d_{n, j}(q)=d_{n}(q)
$$

In other words, the diagram in Fig. 1.2 is commutative.

Even for $q=1$ identity (1.6), which then reads $\sum_{j} d_{n, j}(1)=t_{n}$, is not at all straightforward. It was elegantly proved by Christiane Poupard [Po89] by means of the bivariable generating function

$$
Z(u, v)=1+\sum_{n \geq 1} \sum_{1 \leq l \leq 2 n-1} \frac{u^{2 n-l}}{(2 n-l) !} \frac{v^{l}}{l !} d_{n, l+1}(1) .
$$

She even obtained the following stronger result

$$
Z(u, v)=\frac{\cos ((u-v) / \sqrt{2})}{\cos ((u+v) / \sqrt{2})}
$$

so that

$$
\left.\frac{\partial}{\partial u} Z(u, v)\right|_{\{v=0\}}=\sum_{n \geq 1} \frac{u^{2 n-1}}{(2 n-1) !} d_{n, 2}(1)=\sqrt{2} \tan (u / \sqrt{2}),
$$

which proves $\sum_{j} d_{n, j}(1)=t_{n}$ by appealing to $(P 3)$. 
Finally, she obtains a combinatorial interpretation for the polynomial

$$
d_{n}(s, 1)=\sum_{j} d_{n, j}(1) s^{j}
$$

in terms of strictly ordered binary trees, or in an equivalent manner, of André permutations, which are also alternating (see, e.g. [FS71], [FS71a], [FS73]).

For $q=0$ identity $(1.6)$ reads $\sum_{j} d_{n, j}(0)=C_{n}=\frac{1}{n+1}\left(\begin{array}{c}2 n \\ n\end{array}\right)$. This is a consequence of the identities

$$
\begin{aligned}
d_{n, 2 n}(0) & =\sum_{j} d_{n-1, j}(0) \\
d_{n, j}(0) & =\left(\begin{array}{c}
j-2 \\
n-1
\end{array}\right)-\left(\begin{array}{c}
j-2 \\
n
\end{array}\right)=\frac{2 n-j+1}{n}\left(\begin{array}{c}
j-2 \\
n-1
\end{array}\right)
\end{aligned}
$$

so that, in particular,

$$
d_{n, 2 n}(0)=\frac{1}{n}\left(\begin{array}{c}
2 n-2 \\
n-1
\end{array}\right)=C_{n-1}
$$

easily obtained from $(C 1)$ and $(C 2)$ by induction and iteration, as well as an expression for the generating function

$$
\sum_{n \geq 1} u^{n} \sum_{j} d_{n, j}(0) v^{j}=\frac{1}{1-v-u v}\left(u v^{2}-\frac{v}{2}\left(1-\sqrt{1-4 u v^{2}}\right)\right) .
$$

Again, the reader is referred to the excellent commented bibliography about the sequence A009766 in Sloane's On-Line Encyclopedia of Integer Sequences [S107], in particular the contributions made by David Callan [Cal05] and Emeric Deutsch [De04], where identities (1.11)-(1.14) are actually derived with other initial conditions.

The proof of Theorem 1.1 will be of combinatorial nature. In our previous paper [FH08] we proved that each polynomial $d_{n}(q)$ was a polynomial with positive integral coefficients by showing that it was the generating function for the class $\mathcal{N}_{2 n+1}^{0}$ of permutations called normalized doubloons, by an integral-valued statistic "cmaj" called the compressed major index:

$$
d_{n}(q)=\sum_{\delta \in \mathcal{N}_{2 n+1}^{0}} q^{\mathrm{cmaj} \delta}
$$

In particular, $d_{n}(1)=\# \mathcal{N}_{2 n+1}^{0}=t_{n}$ by (1.4). Normalized doubloon and "cmaj" will be fully described in Section 2. Here we just recall that each normalized doubloon $\delta \in \mathcal{N}_{2 n+1}^{0}$ is a two-row matrix $\delta=$ 


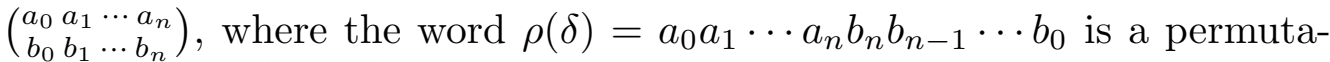
tion of $012 \cdots(2 n+1)$ having further properties that will be given shortly. The set of all normalized doubloons $\delta=\left(\begin{array}{c}a_{0} a_{1} \cdots a_{n} \\ b_{0} b_{1} \cdots b_{n}\end{array}\right)$ such that $b_{0}=j$ is denoted by $\mathcal{N}_{2 n+1, j}^{0}$. It will be shown that $\mathcal{N}_{2 n+1, j}^{0}$ is an empty set for $j=0,1$ and $2 n+1$, so that the subsets $\mathcal{N}_{2 n+1, j}^{0}(j=2,3, \ldots, 2 n)$ form a partition of $\mathcal{N}_{2 n+1}^{0}$. Consequently, the following identity holds:

$$
d_{n}(q)=\sum_{j=2}^{2 n} \sum_{\delta \in \mathcal{N}_{2 n+1, j}^{0}} q^{\mathrm{cmaj} \delta} .
$$

Accorgingly, Theorem 1.1 is a simple corollary of the next theorem.

Theorem 1.2. Let $\left(d_{n, j}(q)\right)$ be the set of polynomials in one variable $q$ defined by $(D 1)-(D 4)$. Then $d_{n, j}(q)$ is the generating polynomial for $\mathcal{N}_{2 n+1, j}^{0}$ by the compressed major index. In other words,

$$
d_{n, j}(q)=\sum_{\delta \in \mathcal{N}_{2 n+1, j}^{0}} q^{\mathrm{cmaj} \delta} .
$$

To prove that the polynomial $d_{n, j}(q)$, as expressed in (1.17), satisfies (D4), symmetry properties must be derived (see Corollaries 4.3, 4.7 and 4.8). This requires a careful geometric study of those doubloons and how the statistic "cmaj" evolves. All this is developed in Sections 3 and 4. The proof of the recurrence is completed in Section 5 and in the final Section concluding remarks are made.

\section{Doubloons}

A doubloon of order $2 n+1$ is a $2 \times(n+1)$-matrix $\delta=\left(\begin{array}{l}a_{0} a_{1} \cdots a_{n} \\ b_{0} b_{1} \cdots b_{n}\end{array}\right)$ such that the word $\rho(\delta)=a_{0} a_{1} \cdots a_{n} b_{n} b_{n-1} \cdots b_{0}$, called the reading of $\delta$, is a permutation of $012 \cdots(2 n+1)$. Let $\mathcal{D}_{2 n+1}\left(\right.$ resp. $\left.\mathcal{D}_{2 n+1}^{0}\right)$ denote the set of all doubloons $\delta=\left(\begin{array}{c}a_{0} a_{1} \cdots a_{n} \\ b_{0} b_{1} \cdots b_{n}\end{array}\right)$ of order $(2 n+1)$ (resp. the subset of all doubloons such that $\left.a_{0}=0\right)$.

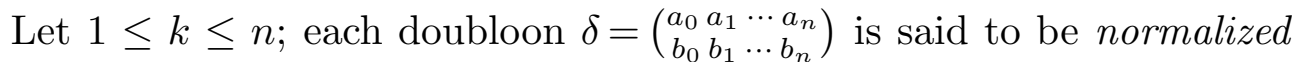
at $k$, if the following two conditions are satisfied:

(N1) exactly one of the two integers $a_{k}, b_{k}$ lies between $a_{k-1}$ and $b_{k-1}$ (we also say that $\delta$ is interlaced at $k$ );

$(N 2)$ either $a_{k-1}>a_{k}$ and $b_{k-1}>b_{k}$, or $a_{k-1}<b_{k}$ and $b_{k-1}<a_{k}$.

In an equivalent manner, $\delta$ is normalized at $k$, if one of the four following orderings holds:

$$
\begin{aligned}
& a_{k-1}<b_{k}<b_{k-1}<a_{k} \\
& b_{k}<b_{k-1}<a_{k}<a_{k-1} \\
& b_{k-1}<a_{k}<a_{k-1}<b_{k} \\
& a_{k}<a_{k-1}<b_{k}<b_{k-1}
\end{aligned}
$$


For each pair of distinct integers $(i, j)$ the symbol $\mathcal{N}_{2 n+1, j}^{i}$ will denote the set of all doubloons $\delta=\left(\begin{array}{ccc}a_{0} a_{1} \cdots & a_{n} \\ b_{0} b_{1} \cdots & \cdots & b_{n}\end{array}\right)$, normalized at every $k=$ $1,2, \ldots, n$, such that $a_{0}=i$ and $b_{0}=j$. The doubloons belonging to $\mathcal{N}_{2 n+1, j}^{0}$ for some $j$ are simply called normalized. Also $\mathcal{N}_{2 n+1}^{0}$ designates the union of the sets $\mathcal{N}_{2 n+1, j}^{0}$ 's. For further results on permutations studied as two-row matrices see [Ha92, Ha94, FH00].

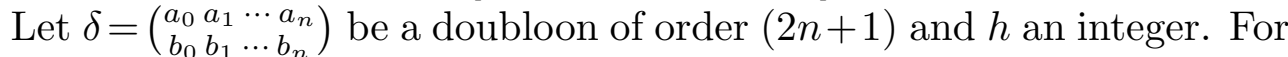
each $k=0,1, \ldots, n$ let $a_{k}^{\prime}=a_{k}+h, b_{k}^{\prime}=b_{k}+h$ be expressed as residues $\bmod (2 n+2)$. The two-row matrix $\left(\begin{array}{cccc}a_{0}^{\prime} & a_{1}^{\prime} & \cdots & a_{n}^{\prime} \\ b_{0}^{\prime} & b_{1}^{\prime} & \cdots & b_{n}^{\prime}\end{array}\right)$, denoted by $\delta+h$, is still a doubloon. Let $T_{h}: \delta \mapsto \delta+h$.

Proposition 2.1. The map $T_{h}$, restricted to $\mathcal{N}_{2 n+1, j}^{i}$, is a bijection onto $\mathcal{N}_{2 n+1, j+h}^{i+h}$ (superscript and subscript being taken $\bmod (2 n+2)$ ).

Proof. The four orderings in (2.1) are cyclic rearrangements of each other, so that if $\delta$ is normalized at each $i$, the doubloon $T_{h} \delta=\delta+h$ has the same property.

The number of descents, des $\delta$, (resp. the major index, maj $\delta$ ) of a

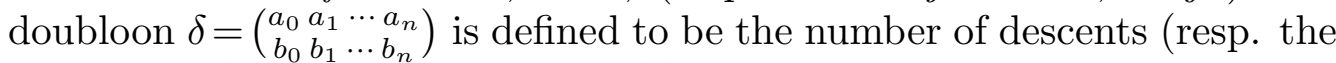
major index) of the permutation $\rho(\delta)=a_{0} a_{1} \cdots a_{n} b_{n} b_{n-1} \cdots b_{0}$, so that if the descents $\left(a_{i}>a_{i+1}\right.$, or $a_{n}>b_{n}$, or still $\left.b_{i-1}>b_{i}\right)$ occur at positions $l_{1}, l_{2}, \ldots, l_{r}$ in $\rho(\delta)$, then $\operatorname{des} \delta=r$ and maj $\delta=l_{1}+l_{2}+\cdots+l_{r}$. The compressed major index, $\operatorname{cmaj} \delta$, of $\delta$ is defined by

$$
\operatorname{cmaj} \delta=\operatorname{maj} \delta-(n+1) \operatorname{des} \delta+\left(\begin{array}{l}
n \\
2
\end{array}\right)
$$

For example, there is one normalized doubloon of order $3(n=1)$ :

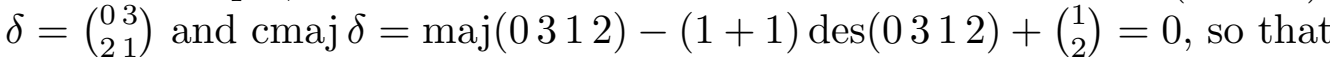
$d_{1,2}(q)=1$. There are four normalized doubloons of order $5(n=2)$ and the partition of $\mathcal{N}_{5}^{0}$ reads:

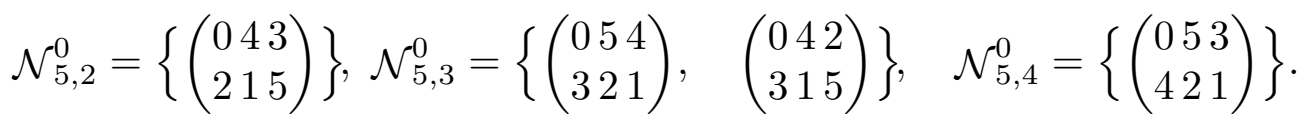

We have $\operatorname{cmaj}(043512)=\operatorname{maj}(043512)-3 \operatorname{des}(043512)+\left(\begin{array}{l}2 \\ 2\end{array}\right)=(2+4)-$ $3 \times 2+1=1$, so that $d_{2,2}(q)=q$. Furthermore, $d_{2,3}(q)=1+q$ and $d_{2,4}(q)=1$, as expected (see Fig. 1.2).

\section{Operations on doubloons}

In this section we study the actions of several operators on the statistic "cmaj." First, we recall the action of the dihedral group on the traditional statistics "des" and "maj," in particular characterize the images of 
each permutation $\sigma=\sigma(1) \sigma(2) \cdots \sigma(n)$ under the reversal $\mathbf{r}$ (resp. complement $\mathbf{c}$ ) that maps $\sigma$ onto its mirror image $\mathbf{r} \sigma=\sigma(n) \cdots \sigma(2) \sigma(1)$ (resp. onto its complement $\mathbf{c} \sigma=(n+1-\sigma(1))(n+1-\sigma(2)) \cdots(n+1-\sigma(n)))$. The next properties are well-known (see, e.g., [FS78]): if $\sigma$ belongs to $\mathfrak{S}_{n}$, then

$$
\begin{aligned}
& \operatorname{des} \mathbf{r} \sigma=n-1-\operatorname{des} \sigma ; \quad \operatorname{maj} \mathbf{r} \sigma=\operatorname{maj} \sigma-n \operatorname{des} \sigma+\left(\begin{array}{l}
n \\
2
\end{array}\right) ; \\
& \operatorname{des} \mathbf{c} \sigma=n-1-\operatorname{des} \sigma ; \quad \operatorname{maj} \mathbf{c} \sigma=\left(\begin{array}{c}
n \\
2
\end{array}\right)-\operatorname{maj} \sigma ; \\
& \operatorname{des} \mathbf{r} \mathbf{c} \sigma=\operatorname{des} \sigma ; \quad \operatorname{maj} \mathbf{r} \mathbf{c} \sigma=n \operatorname{des} \sigma-\operatorname{maj} \sigma .
\end{aligned}
$$

Now let $i, j$ be the two integers defined by $\sigma(1)=n-j, \sigma(n)=n-i$ and $\sigma^{\prime}$ be the permutation mapping $k$ onto

$$
\sigma^{\prime}(k)= \begin{cases}\sigma(k)+i, & \text { if } \sigma(k)+i \leq n \\ \sigma(k)+i-n, & \text { if } \sigma(k)+i>n .\end{cases}
$$

The operation $\sigma \mapsto \sigma^{\prime}$ and Property (3.5) below already appear in [Ha92b] for the study of the $Z$-statistic.

Lemma 3.1. We have:

$$
\begin{aligned}
\operatorname{des} \sigma-\operatorname{des} \sigma^{\prime} & = \begin{cases}0, & \text { if } i<j ; \\
1, & \text { if } i>j ;\end{cases} \\
\operatorname{maj} \sigma-\operatorname{maj} \sigma^{\prime} & =i .
\end{aligned}
$$

Proof. As $\sigma(n)=n-i$, we can factorize the word $\sigma=\sigma(1) \sigma(2) \cdots \sigma(n)$ as a product $p_{0} q_{1} p_{1} \cdots q_{r} p_{r}(r \geq 1)$, where the letters of all $p_{k}$ 's (resp. all $q_{k}$ 's) are smaller than or equal to (resp. greater than) $n-i$. Also, let $p_{0}^{\prime} q_{1}^{\prime} p_{1}^{\prime} \cdots q_{r}^{\prime} p_{r}^{\prime}$ be the corresponding factorization of $\sigma^{\prime}$ such that $\lambda p_{0}^{\prime}=$ $\lambda p_{0}, \lambda q_{1}^{\prime}=\lambda q_{1}, \ldots\left(\lambda\right.$ being the word length). In particular, $p_{r}$ (and $p_{r}^{\prime}$ ) is never empty since $\sigma(n)=n-i$ (and $\left.\sigma^{\prime}(n)=n\right)$, but $p_{0}$ (resp. and $p_{0}^{\prime}$ ) is nonempty if and only if $\sigma(1)+i=n-j+i \leq n$, that is, if $i<j$.

As the rightmost letter of each factor $q_{k}$ is larger than the leftmost letter of $p_{k}$, we have

$$
\operatorname{des} \sigma=\operatorname{des} p_{0}+\operatorname{des} q_{1}+1+\operatorname{des} p_{1}+\cdots+\operatorname{des} q_{r}+1+\operatorname{des} p_{r} .
$$

In $\sigma^{\prime}$ the rightmost letter of each $p_{k}^{\prime}$ is greater than the leftmost letter of $q_{k+1}^{\prime}$, so that, if $i<j$,

$$
\begin{aligned}
\operatorname{des} \sigma^{\prime} & =\operatorname{des} p_{0}^{\prime}+1+\operatorname{des} q_{1}^{\prime}+\cdots+\operatorname{des} p_{r-1}^{\prime}+1+\operatorname{des} q_{r}^{\prime}+\operatorname{des} p_{r}^{\prime} \\
& =\operatorname{des} p_{0}+\operatorname{des} q_{1}+1+\cdots+\operatorname{des} p_{r-1}+\operatorname{des} q_{r}+1+\operatorname{des} p_{r} \\
& =\operatorname{des} \sigma,
\end{aligned}
$$

while, if $i>j$, the factor $p_{0}^{\prime}$ is empty and

$$
\begin{aligned}
\operatorname{des} \sigma^{\prime} & =\operatorname{des} q_{1}^{\prime}+\cdots+\operatorname{des} p_{r-1}^{\prime}+1+\operatorname{des} q_{r}^{\prime}+\operatorname{des} p_{r}^{\prime} \\
& =\operatorname{des} \sigma-1 .
\end{aligned}
$$


However, in both cases we have:

$$
\begin{aligned}
\operatorname{maj} \sigma-\operatorname{maj} \sigma^{\prime}=\lambda\left(p_{0} q_{1}\right)+\lambda\left(p_{0} q_{1} p_{1} q_{2}\right)+\cdots+\lambda\left(p_{0} q_{1} \cdots p_{r-1} q_{r}\right) \\
\quad \quad-\left(\lambda\left(p_{0}^{\prime}\right)+\lambda\left(p_{0}^{\prime} q_{1}^{\prime} p_{1}^{\prime}\right)+\cdots+\lambda\left(p_{0}^{\prime} q_{1}^{\prime} \cdots p_{r-1}^{\prime}\right)\right. \\
=\lambda\left(q_{1}\right)+\lambda\left(q_{2}\right)+\cdots+\lambda\left(q_{r}\right) \\
=\#\{k: \sigma(k)>n-i\}=i .
\end{aligned}
$$

Lemma 3.2. Let $\delta=\left(\begin{array}{c}a_{0} a_{1} \cdots a_{n} \\ b_{0} b_{1} \cdots b_{n}\end{array}\right)$ be a doubloon such that $a_{0}=i$ and $b_{0}=j$. Also, let $\delta^{\prime}=T_{-i}(\delta)=\delta-i=\left(\begin{array}{cccc}0 & a_{1}-i & \cdots & a_{n}-i \\ j-i & b_{1}-i & \cdots & b_{n}-i\end{array}\right)$. Then

$$
\operatorname{cmaj} \delta-\operatorname{cmaj} \delta^{\prime}= \begin{cases}-i, & \text { if } i<j ; \\ n+1-i, & \text { if } i>j .\end{cases}
$$

Proof. Add 1 to each letter of the reading $\rho(\delta)=a_{0} a_{1} \cdots a_{n} b_{n} b_{n-1} \cdots b_{0}$ of $\delta$ and do the same for the reading $\rho\left(\delta^{\prime}\right)$ of $\delta^{\prime}$. We obtain two permutations $\sigma, \sigma^{\prime}$ of $12 \cdots(2 n+2)$ of the form:

$$
\begin{aligned}
& \sigma=(i+1) \sigma(2) \cdots \sigma(2 n+1)(j+1) ; \\
& \sigma^{\prime}=1 \sigma^{\prime}(2) \cdots \sigma^{\prime}(2 n+1)(j-i+1) \text {. }
\end{aligned}
$$

When the transformation $\mathbf{r} \mathbf{c}$ is applied to each of them, we get

$$
\begin{aligned}
\mathbf{r} \mathbf{c} \sigma & =(2 n+2-j) \cdots(2 n+2-i) ; \\
\mathbf{r} \mathbf{c} \sigma^{\prime} & =(2 n+2-j+i) \cdots(2 n+2) .
\end{aligned}
$$

It follows from Lemma 3.1 (with $(2 n+2)$ replacing $n, \mathbf{r} \mathbf{c} \sigma$ instead of $\sigma$ and $\mathbf{r} \mathbf{c} \sigma^{\prime}$ instead of $\left.\sigma^{\prime}\right)$ that

$$
\begin{aligned}
\operatorname{des} \mathbf{r} \mathbf{c} \sigma-\operatorname{des} \mathbf{r} \mathbf{c} \sigma^{\prime} & = \begin{cases}0, & \text { if } i<j ; \\
1, & \text { if } i>j ;\end{cases} \\
\operatorname{maj} \mathbf{r} \mathbf{c} \sigma-\operatorname{maj} \mathbf{r} \mathbf{c} \sigma^{\prime} & =i,
\end{aligned}
$$

so that by $(3.3)$

$$
\begin{aligned}
\operatorname{des} \sigma-\operatorname{des} \sigma^{\prime} & = \begin{cases}0, & \text { if } i<j ; \\
1, & \text { if } i>j ;\end{cases} \\
\operatorname{maj} \sigma-\operatorname{maj} \sigma^{\prime} & =(2 n+2)\left(\operatorname{des} \sigma-\operatorname{des} \sigma^{\prime}\right)-\left(\operatorname{maj} \mathbf{r} \mathbf{c} \sigma-\operatorname{maj} \mathbf{r} \mathbf{c} \sigma^{\prime}\right) \\
& =(2 n+2)\left(\operatorname{des} \sigma-\operatorname{des} \sigma^{\prime}\right)-i
\end{aligned}
$$

As $\operatorname{cmaj} \delta=\operatorname{maj} \sigma-(n+1) \operatorname{des} \sigma+\left(\begin{array}{l}n \\ 2\end{array}\right)$, we get

$$
\begin{aligned}
\operatorname{cmaj} \delta-\operatorname{cmaj} \delta^{\prime} & =\left(\operatorname{maj} \sigma-\operatorname{maj} \sigma^{\prime}\right)-(n+1)\left(\operatorname{des} \sigma-\operatorname{des} \sigma^{\prime}\right) \\
& =(n+1)\left(\operatorname{des} \sigma-\operatorname{des} \sigma^{\prime}\right)-i \\
& = \begin{cases}-i, & \text { if } i<j ; \\
n+1-i, & \text { if } i>j .\end{cases}
\end{aligned}
$$


Lemma 3.3. Let $\delta=\left(\begin{array}{cccc}0 & a_{1} & \cdots & a_{n} \\ b_{0} & b_{1} & \cdots & b_{n}\end{array}\right) \mapsto \delta^{\prime}=\left(\begin{array}{cccc}a_{0}^{\prime} & a_{1}^{\prime} & \cdots & a_{n}^{\prime} \\ b_{0}^{\prime} & b_{1}^{\prime} & \cdots & b_{n}^{\prime}\end{array}\right)$ be the transformation mapping $\delta \in \mathcal{D}_{2 n+1}^{0}$ onto the doubloon $\delta^{\prime}$ obtained from $\delta$ by replacing each entry $a_{k}\left(\right.$ resp. $\left.b_{k}\right)$ by $a_{k}^{\prime}=(2 n+1)-a_{k}\left(\right.$ resp. $\left.b_{k}^{\prime}=(2 n+1)-b_{k}\right)$. Then $\operatorname{cmaj} \delta=n(n-1)-\operatorname{cmaj} \delta^{\prime}$.

Proof. Again, add 1 to each element of $\rho(\delta)$ and of $\rho\left(\delta^{\prime}\right)$ to obtain two permutations $\sigma$ and $\sigma^{\prime}$ of $12 \cdots(2 n+2)$. We have: $\sigma^{\prime}=\mathbf{c} \sigma$. By (3.2) $\operatorname{des} \sigma+\operatorname{des} \sigma^{\prime}=2 n+1$ and maj $\sigma+\operatorname{maj} \sigma^{\prime}=\left(\begin{array}{c}2 n+2 \\ 2\end{array}\right)=(n+1)(2 n+1)$. Hence, $\operatorname{cmaj} \delta+\operatorname{cmaj} \delta^{\prime}=\operatorname{maj} \sigma+\operatorname{maj} \sigma^{\prime}-(n+1)\left(\operatorname{des} \sigma+\operatorname{des} \sigma^{\prime}\right)+n(n-1)=$ $(n+1)(2 n+1)-(n+1)(2 n+1)+n(n-1)=n(n-1)$.

Let $\Gamma:\left(\begin{array}{ccc}0 & a_{1} \cdots a_{n} \\ b_{0} b_{1} \cdots b_{n}\end{array}\right) \mapsto\left(\begin{array}{cc}0 & 2 n+2-a_{1} \cdots 2 n+2-a_{n} \\ 2 n+2-b_{0} & 2 n+2-b_{1} \cdots 2 n+2-b_{n}\end{array}\right)\left[\Gamma: \delta \mapsto \delta^{\prime \prime}\right.$ in short $]$ be the transformation mapping each doubloon $\delta=\left(\begin{array}{cccc}0 & a_{1} & \cdots & a_{n} \\ b_{0} & b_{1} & \cdots & b_{n}\end{array}\right) \in \mathcal{D}_{2 n+1}^{0}$

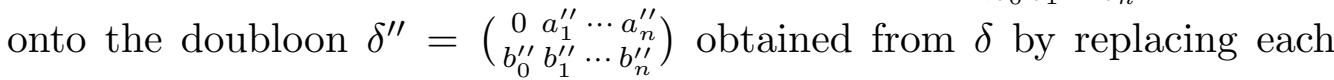
entry $a_{k}\left(\right.$ resp. $\left.b_{k}\right)$ by the residue $a_{k}^{\prime \prime}=(2 n+2)-a_{k}\left(\right.$ resp. $b_{k}^{\prime \prime}=(2 n+$ 2) $\left.-b_{k}\right)$.

Remark. If $\delta$ is interlaced at each $k$ (condition $(N 1)$ holds at each $k$ ), the same property holds for $\Gamma(\delta)$. However, if $\delta$ is normalized at each $k$, the property is not preserved under the transformation $\Gamma$.

Lemma 3.4. For each $\delta \in \mathcal{D}_{2 n+1}^{0}$ we have: $\operatorname{cmaj} \delta+\operatorname{cmaj} \Gamma(\delta)=n^{2}$.

Proof. First, transform $\delta$ into the doubloon $\delta^{\prime}$ defined in Lemma 3.3, so that $\delta^{\prime}=\left(\begin{array}{cccc}2 n+1 & a_{1}^{\prime} & \cdots & a_{n}^{\prime} \\ 2 n+1-j & b_{1}^{\prime} & \cdots & b_{n}^{\prime}\end{array}\right)$ and cmaj $\delta=n(n-1)-$ cmaj $\delta^{\prime}$. Next, apply Lemma 3.2 to $\delta^{\prime}$ with $i=2 n+1$, so that the new doubloon is of the form $\delta^{\prime \prime}=\left(\begin{array}{cccc}0 & a_{1}^{\prime \prime} & \cdots & a_{n}^{\prime \prime} \\ 2 n+2-j & b_{1}^{\prime \prime} & \cdots & b_{n}^{\prime \prime}\end{array}\right)$. Hence, cmaj $\delta^{\prime}-\operatorname{cmaj} \delta^{\prime \prime}=(n+1)-(2 n+1)=-n$ and $\operatorname{cmaj} \delta=n(n-1)-\operatorname{cmaj} \delta^{\prime \prime}+n=n^{2}-\operatorname{cmaj} \delta^{\prime \prime}$.

\section{Further operations on doubloons}

In our previous paper [FHa08] we also introduced a class of transformations $\phi_{i}(0 \leq i \leq n)$ on $\mathcal{D}_{2 n+1}$, called micro flips, which permute the entries in a given column. By definition,

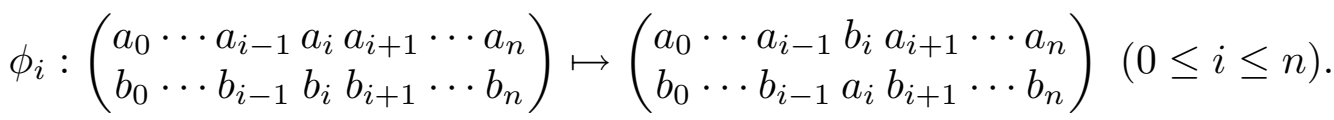

Next, the macro flips $\Phi_{i}$ are defined by $\Phi_{i}=\phi_{i} \phi_{i+1} \cdots \phi_{n}(1 \leq i \leq n)$. Note that both $\phi_{i}$ and $\Phi_{i}$ are involutions of $\mathcal{D}_{2 n+1}$. In particular,

$$
\Phi_{1} \Phi_{2} \cdots \Phi_{i}=\prod_{\substack{j \text { odd } \\ j \leq i}} \phi_{j} .
$$

By means of the transformation $\Gamma$ (see Lemma 3.4 above) and the involutions $\Phi_{i}$ 's we now construct a bijection of $\mathcal{N}_{2 n+1, j}^{0}$ onto $\mathcal{N}_{2 n+1,2 n+2-j}^{0}$. 
Lemma 4.1. Let $\delta=\left(\begin{array}{ccc}0 & a_{1} \cdots a_{n} \\ b_{0} b_{1} \cdots b_{n}\end{array}\right)$ be a normalized doubloon and let $\Gamma(\delta)=\delta^{\prime \prime}=\left(\begin{array}{ccc}0 & a_{1}^{\prime \prime} \cdots & a_{n}^{\prime \prime} \\ b_{0}^{\prime \prime} & b_{1}^{\prime \prime} \cdots & b_{n}^{\prime \prime}\end{array}\right)$. Then, for each $i=1,2, \ldots, n$ the doubloon $\Phi_{1} \Phi_{2} \cdots \Phi_{i}\left(\delta^{\prime \prime}\right)$ is normalized at each integer $1,2, \ldots, i$. In particular, $\Phi_{1} \Phi_{2} \cdots \Phi_{n}\left(\delta^{\prime \prime}\right)$ is normalized.

Proof. Using (4.1) we have:

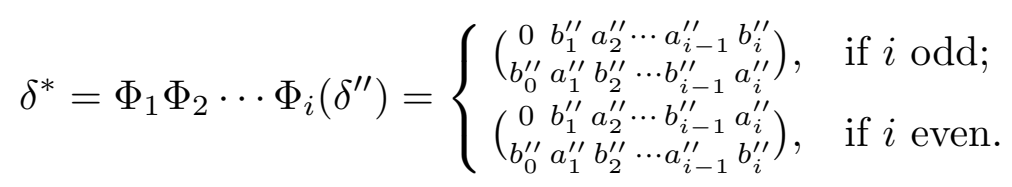

The doubloon $\delta^{\prime \prime}$ is not normalized (see the Remark before Lemma 3.4), but is interlaced at each $i$, so that the relations $a_{j-1}^{\prime \prime}<a_{j}^{\prime \prime}, b_{j-1}^{\prime \prime}<b_{j}^{\prime \prime}$ or $a_{j-1}^{\prime \prime}>a_{j}^{\prime \prime}, b_{j-1}^{\prime \prime}>b_{j}^{\prime \prime}$ hold for all $j$. This shows that $\delta^{*}$ is normalized whenever $j$ is odd or even.

For the next Proposition we need the following property proved in our previous paper [FHa08, Theorem 3.5]:

(4.2) Let $\delta=\left(\begin{array}{cccc}0 & a_{1} & \cdots & a_{n} \\ b_{0} & b_{1} & \cdots & b_{n}\end{array}\right)$ be an interlaced doubloon, normalized at $i$. Then $\operatorname{cmaj} \Phi_{i}(\delta)-\operatorname{cmaj} \delta=n-i+1 \quad(1 \leq i \leq n)$.

Proposition 4.2. The transformation $\Phi_{1} \Phi_{2} \cdots \Phi_{n} \Gamma: \delta \mapsto \delta^{*}$ is a bijection of $\mathcal{N}_{2 n+1, j}^{0}$ onto $\mathcal{N}_{2 n+1,2 n+2-j}^{0}$ having the property

$$
\operatorname{cmaj} \delta+\operatorname{cmaj} \delta^{*}=\left(\begin{array}{l}
n \\
2
\end{array}\right) .
$$

Proof. Let $\delta^{\prime \prime}=\Gamma(\delta)$. As $\Phi_{1} \Phi_{2} \cdots \Phi_{i}\left(\delta^{\prime \prime}\right)$ is normalized at $i$, we have

$$
\operatorname{cmaj} \Phi_{i} \Phi_{1} \Phi_{2} \cdots \Phi_{i}\left(\delta^{\prime \prime}\right)-\operatorname{cmaj} \Phi_{1} \Phi_{2} \cdots \Phi_{i}\left(\delta^{\prime \prime}\right)=n-i+1
$$

by (4.2). Summing over all $i=1,2, \ldots, n$ we get

$$
\begin{aligned}
\sum_{i=1}^{n}\left(\operatorname{cmaj} \Phi_{1} \Phi_{2} \cdots \Phi_{i-1}\left(\delta^{\prime \prime}\right)-\operatorname{cmaj} \Phi_{1} \Phi_{2} \cdots \Phi_{i}\left(\delta^{\prime \prime}\right)\right) & =\sum_{i=1}^{n}(n-i+1) \\
\operatorname{cmaj} \delta^{\prime \prime}-\operatorname{cmaj} \Phi_{1} \Phi_{2} \cdots \Phi_{n}\left(\delta^{\prime \prime}\right) & =n^{2}-\left(\begin{array}{c}
n \\
2
\end{array}\right) .
\end{aligned}
$$

Using Lemma 3.4 we conclude that $\left(n^{2}-\operatorname{cmaj} \delta\right)-\operatorname{cmaj} \delta^{*}=n^{2}-\left(\begin{array}{c}n \\ 2\end{array}\right)$ and then $\operatorname{cmaj} \delta+\operatorname{cmaj} \delta^{*}=\left(\begin{array}{l}n \\ 2\end{array}\right)$.

Example. For $n=2$ we have $\Phi_{1} \Phi_{2}=\phi_{1}$. The four normalized doubloons of order $5(n=2)$ displayed at the end of Section 2 are mapped under $\Phi_{1} \Phi_{2} \Gamma=\phi_{1} \Gamma$ as shown in Fig. 4.1. Next to each doubloon appears the value of its "cmaj". 


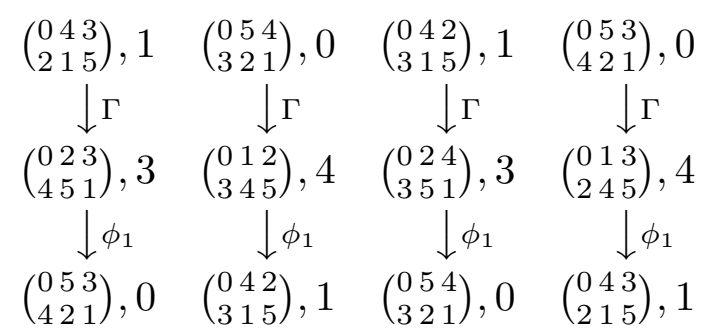

Fig. 4.1. The transformation $\Phi_{1} \Phi_{2} \cdots \Phi_{n} \Gamma$

Let $d_{n, j}(q)=\sum_{\delta \in \mathcal{N}_{2 n+1, j}^{0}} q^{\text {cmaj } \delta}$. The final purpose is to show that $d_{n, j}(q)$ satisfies relations $(D 1)-(D 4)$. This will be done in Section 5 . In the rest of this section we state some symmetry properties of the $d_{n, j}(q)$ 's.

Corollary 4.3. For $2 \leq j \leq 2 n$ we have:

$$
d_{n, j}(q)=q^{\left(\begin{array}{c}
n \\
2
\end{array}\right)} d_{n, 2 n+2-j}\left(q^{-1}\right) .
$$

Proof. This follows from the previous proposition:

$$
d_{n, j}(q)=\sum_{\delta \in \mathcal{N}_{2 n+1, j}^{0}} q^{\mathrm{cmaj} \delta}=\sum_{\delta^{*} \in \mathcal{N}_{2 n+1,2 n+1-j}^{0}} q^{\left(\begin{array}{c}
n \\
2
\end{array}\right)-\mathrm{cmaj} \delta^{*}}=q^{\left(\begin{array}{l}
n \\
2
\end{array}\right)} d_{n, 2 n+2-j}\left(q^{-1}\right) .
$$

In the next lemma we study the action of the sole transposition $\phi_{0}$ that permutes the leftmost entries of each doubloon.

Lemma 4.4. For each normalized doubloon $\delta$

$$
\operatorname{cmaj} \delta-\operatorname{cmaj} \phi_{0}(\delta)=-n
$$

Proof. Let $\rho(\delta)=0 a_{1} \cdots a_{n} b_{n} \cdots b_{1} b_{0}$ be the reading of $\delta$, so that $\rho\left(\phi_{0}(\delta)\right)=b_{0} a_{1} \cdots a_{n} b_{n} \cdots b_{1} 0$. As $\delta$ is normalized (and, in particular, interlaced), we have: $0<b_{1}<b_{0}<a_{1}$. Thus, $\rho(\delta)$ starts and ends with a rise $\left(0<a_{1}\right.$ and $\left.b_{1}<a_{0}\right)$, while $\rho\left(\phi_{0}(\delta)\right)$ starts with a rise $b_{0}<a_{1}$ and ends with a descent $b_{1}>0$. Hence, $\operatorname{des} \rho(\delta)=\operatorname{des} \rho\left(\phi_{0}(\delta)\right)-1$ and $\operatorname{maj} \rho(\delta)=\operatorname{maj} \rho\left(\phi_{0}(\delta)\right)-(2 n+1)$ and $\operatorname{cmaj} \delta-\operatorname{cmaj} \rho\left(\phi_{0}(\delta)\right)=-(2 n+$ 1) $-(n+1)-1=-n$

The involution $\phi_{0} \Phi_{1}=\Phi_{1} \phi_{0}=\phi_{0} \phi_{1} \cdots \phi_{n}$ transposes the two rows of each doubloon $\delta \in \mathcal{D}_{2 n+1}$.

Lemma 4.5. For each normalized doubloon $\delta$

$$
\operatorname{cmaj} \delta=\operatorname{cmaj} \phi_{0} \Phi_{1}(\delta)
$$


Proof. First, $\operatorname{cmaj} \delta=\operatorname{cmaj} \phi_{0}(\delta)-n$ by the previous lemma. Furthermore, as $\phi_{0} \Phi_{1}(\delta)$ remains normalized at 1, relation (4.2) implies that $\operatorname{cmaj} \Phi_{1}\left(\phi_{0} \Phi_{1}(\delta)\right)-\operatorname{cmaj} \phi_{0} \Phi_{1}(\delta)=n-1+1$. Thus,

$$
\begin{aligned}
\operatorname{cmaj} \phi_{0} \Phi_{1}(\delta) & =\operatorname{cmaj} \phi_{0}(\delta)-n \\
& =\operatorname{cmaj} \delta+n-n=\operatorname{cmaj} \delta .
\end{aligned}
$$

We next study the joint action of the operators $T_{-j}$ (introduced in Lemma. 3.2) $\phi_{0}$ and $\Phi_{1}$.

Proposition 4.6. The transformation $T_{-j} \phi_{0} \Phi_{1}: \delta \mapsto \delta^{\prime}$ is a bijection of $\mathcal{N}_{2 n+1, j}^{0}$ onto $\mathcal{N}_{2 n+1,2 n+2-j}^{0}$ having the property that

$$
\operatorname{cmaj} \delta-\operatorname{cmaj} \delta^{\prime}=n+1-j .
$$

Proof. Let $\delta \in \mathcal{N}_{2 n+1, j}^{0}$. Then, $\phi_{0} \Phi_{1}(\delta)$ is normalized at each $i=$ $1,2, \ldots, n$ and also $T_{-j} \phi_{0} \Phi_{1}(\delta)$ by the remark made before Lemma 3.2. Thus, $\delta^{\prime} \in \mathcal{N}_{2 n+1,2 n+2-j}^{0}$ and the map $\delta \mapsto \delta^{\prime}$ is bijective. Furthermore, $\operatorname{cmaj} \delta=\operatorname{cmaj} \phi_{0} \Phi_{1}(\delta)$ by Lemma 4.5 and $\operatorname{cmaj} \phi_{0} \Phi_{1}(\delta)=\operatorname{cmaj} \delta^{\prime}+n+$ $1-j$ by Lemma 3.2 .

Example. Again consider the four normalized doubloons of order 5 . We get the display of Fig. 4.2. with the value of "cmaj" next to each doubloon.

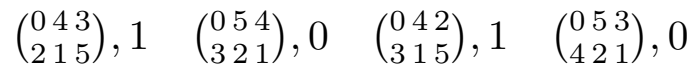

$$
\begin{aligned}
& \downarrow \phi_{0} \Phi_{1} \quad \downarrow \phi_{0} \Phi_{1} \quad \downarrow \phi_{0} \Phi_{1} \quad \downarrow \phi_{0} \Phi_{1} \\
& \left(\begin{array}{lll}
2 & 1 & 5 \\
0 & 4 & 3
\end{array}\right), 1 \quad\left(\begin{array}{lll}
3 & 2 & 1 \\
0 & 5 & 4
\end{array}\right), 0 \quad\left(\begin{array}{lll}
3 & 1 & 5 \\
0 & 4 & 2
\end{array}\right), 1 \quad\left(\begin{array}{lll}
4 & 2 & 1 \\
0 & 5 & 3
\end{array}\right), 0 \\
& \downarrow T_{-2} \quad \downarrow T_{-3} \quad \downarrow T_{-3} \quad \downarrow T_{-4} \\
& \left(\begin{array}{lll}
0 & 5 & 3 \\
4 & 2 & 1
\end{array}\right), 0 \quad\left(\begin{array}{lll}
0 & 5 & 4 \\
3 & 2 & 1
\end{array}\right), 0 \quad\left(\begin{array}{lll}
0 & 4 & 2 \\
3 & 1 & 5
\end{array}\right), 1 \quad\left(\begin{array}{lll}
0 & 4 & 3 \\
2 & 1 & 5
\end{array}\right), 1
\end{aligned}
$$

Fig. 4.2. The transformation $T_{-j} \phi_{0} \Phi_{1}$

Corollary 4.7. For $2 \leq j \leq 2 n$ we have:

$$
d_{n, j}(q)=q^{n+1-j} d_{n, 2 n+2-j}(q) .
$$

The corollary immediately follows from Proposition 4.6. In its turn the next corollary is a consequence of both Corollaries 4.3 and 4.7.

Corollary 4.8. For $2 \leq j \leq 2 n$ we have:

$$
d_{n, j}(q)=q^{\left(\begin{array}{c}
n+1 \\
2
\end{array}\right)+1-j} d_{n, j}\left(q^{-1}\right) .
$$

As could be seen in Fig. 1.2 for the first values, the polynomial $d_{n, j}(q)$ is a multiple of $d_{n, 2 n+2-j}(q)$ (Corollary 4.7) and Corollary 4.8 indicates a symmetry between its coefficients. 


\section{The recurrence itself}

In this section we prove Theorem 1.2 , that is, we show that relations $(D 1)-(D 4)$ hold for $d_{n, j}(q)=\sum_{\delta \in \mathcal{N}_{2 n+1, j}^{0}} q^{\text {cmaj } \delta}$. The first two relations $(D 1)-(D 2)$ are evidently true. For proving that relations $(D 3)-(D 4)$ hold for such a $d_{n, j}(q)$ we start with a doubloon $\delta \in \mathcal{N}_{2 n+1}^{0}$, drop its leftmost column and compare the compressed major indices of $\delta$ and of the doubloon obtained after deletion. In so doing we get the following result.

Lemma 5.1. To each doubloon $\delta \in \mathcal{N}_{2 n+1, j}^{0}$ there corresponds a unique triplet $\left(k, l, \delta^{\prime}\right)$ such that $k \geq j-1, l \leq j-2$ and $\delta^{\prime} \in \mathcal{N}_{2 n-1, l}^{k}$ having the property that

$$
\operatorname{cmaj} \delta=\operatorname{cmaj} \delta^{\prime}+(n-1) .
$$

Proof. Let $\delta=\left(\begin{array}{cccc}0 & a_{1} & \cdots & a_{n} \\ j & b_{1} & \cdots & b_{n}\end{array}\right) \in \mathcal{N}_{2 n+1, j}^{0}$ and define

$$
a_{i}^{\prime}= \begin{cases}a_{i}-1, & \text { if } a_{i}<j \\ a_{i}-2, & \text { if } j<a_{i} \leq 2 n+1\end{cases}
$$

with an analogous definition for the $b_{i}^{\prime}$, the $b$ 's replacing the $a$ 's. Then, $\delta^{\prime}=\left(\begin{array}{lll}a_{1}^{\prime} & \ldots & a_{n}^{\prime} \\ b_{1}^{\prime} & \ldots & b_{n}^{\prime}\end{array}\right)$ is a normalized doubloon of order $(2 n+1)$, but its left-top corner $a_{1}^{\prime}$ is not necessarily 0 . Call it the reduction of $\delta$. As $a_{1}>j>b_{1}$, we see that $b_{1}^{\prime}=b_{1}-1<j-1$ and $a_{1}^{\prime}=a_{1}-2>j-2$, that is, $b_{1}^{\prime} \leq j-2<j-1 \leq a_{1}^{\prime}$. Thus $\delta^{\prime} \in \mathcal{N}_{2 n-1, l}^{k}$ with $k \geq j-1$ and $l \leq j-2$. Conversely, given the triplet $\left(\delta^{\prime}, k, l\right)$ with the above properties, we can uniquely reconstruct the normalized doubloon $\delta$.

As $\delta$ is normalized at 1 , the inequalities $0<b_{1}<j<a_{1}$ hold, so that $\rho(\delta)$ starts and ends with a rise: $0<a_{1}, b_{1}<j$. In particular, $\operatorname{des} \rho(\delta)=\operatorname{des} \rho\left(\delta^{\prime}\right)$. However, maj $\rho(\delta)=\operatorname{maj} \rho\left(\delta^{\prime}\right)+\operatorname{des} \delta$, as the first letter 0 is dropped when going from $\rho(\delta)$ to $\rho\left(\delta^{\prime}\right)$. Hence, $\operatorname{cmaj} \delta-\operatorname{cmaj} \delta^{\prime}=$ $\operatorname{des} \delta-((n+1)-n) \operatorname{des} \delta+\left(\begin{array}{c}n \\ 2\end{array}\right)-\left(\begin{array}{c}n-1 \\ 2\end{array}\right)=n-1$.

The previous Lemma yields the first passage from the generating polynomial $d_{n, j}(q)$ for the normalized doubloons $\delta \in \mathcal{N}_{2 n+1, j}^{0}$ by "cmaj" to the generating polynomial for doubloons of order $(2 n-1)$, as expressed in the next Corollary.

Corollary 5.2. For $2 \leq j \leq 2 n$ we have:

$$
d_{n, j}(q)=q^{n-1} \sum_{k \geq j-1, l \leq j-2} q^{n-k} d_{n-1,2 n+l-k}(q) .
$$


Proof. By the previous Lemma we can write:

$$
d_{n, j}(q)=\sum_{\delta \in \mathcal{N}_{2 n+1, j}^{0}} q^{\mathrm{cmaj} \delta}=q^{n-1} \sum_{k \geq j-1, l \leq j-2} \sum_{\delta^{\prime} \in \mathcal{N}_{2 n-1, l}^{k}} q^{\mathrm{cmaj} \delta^{\prime}} .
$$

With each $\delta^{\prime} \in \mathcal{N}_{2 n-1, l}^{k}$ associate $\delta^{\prime \prime}=T_{-k} \delta^{\prime}=\delta^{\prime}-k$. By Lemma 3.2 we get $\delta^{\prime \prime} \in \mathcal{N}_{2 n-1,2 n+l-k}^{0}$ (note that the residue $\bmod (2 n+2)$ must be considered in the subscript for $l<k$ ) and $\operatorname{cmaj} \delta^{\prime}-\operatorname{cmaj} \delta^{\prime \prime}=n-k$. Hence,

$$
\sum_{\delta^{\prime} \in \mathcal{N}_{2 n-1, l}^{k}} q^{\mathrm{cmaj} \delta^{\prime}}=q^{n-k} \sum_{\delta^{\prime \prime} \in \mathcal{N}_{2 n-1,2 n+l-k}^{0}} q^{\mathrm{cmaj} \delta^{\prime \prime}}=q^{n-k} d_{n-1,2 n+l-k}(q) .
$$

When $j=2$ in (5.2), we get:

$$
\begin{aligned}
d_{n, 2}(q) & =\sum_{k \geq 2} q^{2 n-k-1} d_{n-1,2 n-k}(q) \\
& =\sum_{k \geq 2} q^{i-1} d_{n-1, i}(q) \quad[\text { by the change of variables } i=2 n-k \text { ] }
\end{aligned}
$$

and also

$$
d_{n, 2}(q)=q^{n-1} \sum_{i \geq 2} d_{n-1, i}(q) \quad \text { [by using (4.7).] }
$$

Consequently, relation $(D 3)$ holds for the polynomial $d_{n, j}(q)=\sum q^{\mathrm{cmaj} \delta}$.

Using (5.2) it is also easy to derive the identity: $\delta \in \mathcal{N}_{2 n+1, j}^{0}$

$$
q d_{n, 3}(q)=(q+1) d_{n, 2}(q) .
$$

Proposition 5.2. For $2 \leq j \leq 2 n$ we have:

$$
d_{n, j}(q)=\sum_{i} \frac{q^{\max (0, i+1-j)}-q^{\min (i, 2 n+1-j)}}{1-q} d_{n-1, i}(q) .
$$

Proof. Let $i=2 n+l-k$ with $0 \leq l \leq j-2$ and $j-1 \leq k \leq 2 n-1$. This implies $0 \leq i+k-2 n \leq j-2$, or still $2 n-i \leq k \leq 2 n+j-i-2$. Taking the two relations keeping $k$ within bounds into account we get the double inequality:

$$
\max (2 n-i, j-1) \leq k \leq \min (2 n+j-i-2,2 n-1) .
$$

Identity (5.2) may then be rewritten as

$$
d_{n, j}(q)=q^{2 n-1} \sum_{i} d_{2 n-1, i}(q) \sum_{k} q^{-k},
$$


with $k$ ranging over the interval defined in (5.4). The geometric sum over $k$ is equal to

$$
\frac{q^{-\max (2 n-i, j-1)}-q^{-\min (2 n+j-i-2,2 n-1)-1}}{1-q^{-1}} .
$$

Now, $2 n-\max (2 n-i, j-1)=2 n+\min (-2 n+i,-j+1)=\min (i, 2 n-j+1)$ and $2 n-1-\min (2 n+j-i-2,2 n-1)=\max (0,-j+i+1)$. Accordingly,

$$
q^{2 n-1} \sum_{k \text { subject to }(5.4)} q^{-k}=\frac{q^{\max (0, i+1-j)}-q^{\min (i, 2 n+1-j)}}{1-q} .
$$

This proves (5.3) when reporting the latter expression into (5.5).

For getting rid of "max" and "min" from identity (5.3) we decompose the sum into four subsums, assuming $j \geq 3$ (when $j=2$ identity (5.3) gives back $(D 3))$. We obtain:

$$
\begin{aligned}
(1-q) d_{n, j}(q)= & \sum_{i=0}^{j-1} d_{n-1, i}(q)+\sum_{i=j}^{2 n+1} q^{i+1-j} d_{n-1, i}(q) \\
& \quad-\sum_{i=0}^{2 n-j} q^{i} d_{n-1, i}(q)-\sum_{i=2 n-j+1}^{2 n+1} q^{2 n+1-j} d_{n-1, i}(q) .
\end{aligned}
$$

By means of $(5.6)$ we calculate $d_{n, j}(q)-d_{n, j-1}(q)$ and then $-d_{n, j-1}(q)+$ $d_{n, j-2}(q)$, whose sum is the left-hand side of $(D 4)$. In the computation of $(1-q)\left(d_{n, j}(q)-d_{n, j-1}(q)\right)$ the contribution of the first subsum is simply $d_{n-1, j-1}(q)$. Next, $q^{2 n-j+1} d_{n-1,2 n-j+1}(q)$ is the contribution of the second subsum. For the third subsum we get

$$
-q d_{n-1, j-1}(q)+\sum_{i=j}^{2 n+1} q^{i-j+1}(1-q) d_{n-1, i}(q)
$$

and for the fourth one

$$
-q^{2 n+1-j} d_{n-1,2 n+1-j}(q)+\sum_{i=2 n-j+2}^{2 n+1} q^{2 n+1-j}(q-1) d_{n-1, i}(q) .
$$

Altogether

$$
\begin{aligned}
\text { (5.7) } & d_{n, j}(q)-d_{n, j-1}(q) \\
& =d_{n-1, j-1}(q)+\sum_{i=j}^{2 n+1} q^{i-j+1} d_{n-1, i}(q)-\sum_{i=2 n-j+2}^{2 n+1} q^{2 n+1-j} d_{n-1, i}(q) ; \\
(5.8) \quad & -d_{n, j-1}(q)+d_{n, j-2}(q) \\
= & -d_{n-1, j-2}(q)-\sum_{i=j-1}^{2 n+1} q^{i-j+2} d_{n-1, i}(q)+\sum_{i=2 n-j+3}^{2 n+1} q^{2 n+2-j} d_{n-1, i}(q) .
\end{aligned}
$$


Summing (5.7) and (5.8) we get:

$$
\begin{aligned}
& d_{n, j}(q)-2 d_{n, j-1}(q)+d_{n-1, j-2}(q) \\
& =d_{n-1, j-1}(q)-d_{n-1, j-2}(q) \\
& \quad-q d_{n-1, j-1}(q)+(1-q) \sum_{i=j}^{2 n+1} q^{i-j+1} d_{n-1, i}(q) \\
& \quad-q^{2 n+1-j} d_{n-1,2 n-j+2}(q)-(1-q) \sum_{i=2 n-j+3}^{2 n+1} q^{2 n+1-j} d_{n-1, i}(q) .
\end{aligned}
$$

Now, $-q^{2 n+1-j} d_{n-1,2 n-j+2}(q)=-q^{n-1} d_{n-1, j-2}(q)$ by Corollary 4.7. Also

$$
\begin{aligned}
\sum_{i=2 n-j+3}^{2 n+1} q^{2 n+1-j} d_{n-1, i}(q) & =\sum_{i=2 n-j+3}^{2 n-1} q^{2 n+1-j} d_{n-1, i}(q) \\
& =\sum_{k=1}^{j-3} q^{2 n+1-j} d_{n-1,2 n-k}(q) \quad[k=2 n-i] \\
& =\sum_{i=1}^{j-3} q^{n+i+1-j} d_{n-1, i}(q) . \quad[\text { by Corollary } 4.7]
\end{aligned}
$$

Hence, $d_{n, j}(q)-2 d_{n, j-1}(q)+d_{n, j-2}(q)$

$$
\begin{aligned}
= & -(1-q) \sum_{i=1}^{j-3} q^{n+i+1-j} d_{n-1, i}(q) \\
& -\left(1+q^{n-1}\right) d_{n-1, j-2}(q)+(1-q) \sum_{i=j-1}^{2 n-1} q^{i-j+1} d_{n-1, i}(q)
\end{aligned}
$$

for $n \geq 2$ and $3 \leq j \leq 2 n$, which is precisely relation $(D 4)$.

\section{Concluding remarks}

As mentioned in the Introduction, there has been a great number of papers dealing with the Catalan Triangle (the numbers $d_{n, j}(0)$ ). The recurrence for the numbers $d_{n, j}(1)$, namely the set of conditions $(P 1)-(P 4)$, is definitely due to Christiane Poupard [Po89] and was recently rediscovered by Graham and Zang [GZ08]. More exactly, the latter authors introduced the notion of split-pair arrangement. To show that the number of such arrangements of order $n$ was equal to the reduced tangent number $t_{n}$ they set up an algebra for the coefficients $d_{n, j}(1)$ and again produced the recurrence $(P 1)-(P 4)$, but their proof of the identity $\sum_{j} d_{n, j}(1)=t_{n}$ was more elaborate than the original one made by Christiane Poupard [Po89], shortly sketched in the Introduction.

Referring to Section 4 we say that two doubloons $\delta, \delta^{\prime} \in \mathcal{D}_{2 n+1}$ are equivalent if there is a sequence $\phi_{i_{1}}, \phi_{i_{2}}, \ldots, \phi_{i_{k}}$ of micro flips such that 
$\delta^{\prime}=\phi_{i_{1}} \phi_{i_{2}} \cdots \phi_{i_{k}}(\delta)$. A doubloon $\delta=\left(\begin{array}{ccc}a_{0} a_{1} \cdots a_{n} \\ b_{0} b_{1} \cdots b_{n}\end{array}\right)$ is said to be minimal, if $a_{k}<b_{k}$ holds for every $k$. Clearly, every equivalence class of doubloons contains one and only one minimal doubloon. Also, as proved in [FH08], each doubloon $\delta \in \mathcal{D}_{2 n+1}^{0}$ is equivalent to one and only one normalized doubloon.

The link between split-pair arrangements and equivalence classes of interlaced doubloons is the following: start with a interlaced and minimal doubloon $\delta=\left(\begin{array}{ccc}a_{0} & a_{1} \cdots a_{n} \\ b_{0} & b_{1} \cdots & b_{n}\end{array}\right)$ and define the word $w=x_{1} x_{2} \cdots x_{2 n}$ by $x_{l+1}=l$ if and only if $a_{k}$ or $b_{k}$ is equal to $l$. For instance,

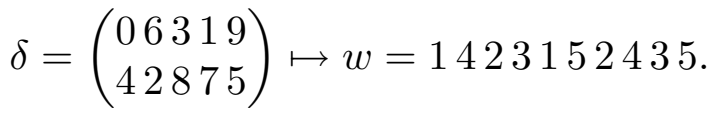

As $\delta$ is interlaced (condition $(N 1)$ ), exactly one of the integers $a_{k}, b_{k}$ lies between $a_{k-1}$ and $b_{k-1}$. If $a_{k}=l<l^{\prime}=b_{k}$, then $x_{l+1}=x_{l^{\prime}+1}=k$. If $a_{k-1}=m<a_{k}=l<b_{k-1}=m^{\prime}<b_{k}=l^{\prime}$, then $x_{m+1}=x_{m^{\prime}+1}=k-1$ and there is exactly one letter equal to $k$, namely $x_{l+1}$ between the two occurrences of $(k-1)$, namely $x_{m+1}$ and $x_{m^{\prime}+1}$. Same conclusion if $a_{k-1}<$ $a_{k}<b_{k-1}<b_{k}$. Those words $w$ of length $2 n$ having the property that exactly one letter equal to $k$ lies between two occurrences of $(k-1)$ for each $k=2,3, \ldots, n$ was called split-pair arrangements by Graham and Zang [GZ08]. The mapping $\delta \mapsto w$ provides a bijection between equivalence classes of interlaced doubloons and those arrangements.

A permutation $\sigma=\sigma(1) \sigma(2) \cdots \sigma(2 n+1)$ of $12 \cdots(2 n+1)$ is said to be alternating if $\sigma(2 i)<\sigma(2 i-1), \sigma(2 i+1)$ for each $i=1,2, \ldots, n$. For each even integer $2 i(1 \leq i \leq n)$ let $w_{i}^{\prime}$ (resp. $\left.w_{i}^{\prime \prime}\right)$ be the longest right factor of $\sigma(1) \cdots \sigma(2 i-1)$ (resp. longest left factor of $\sigma(2 i+1) \cdots \sigma(2 n+1)$ ), all letters of which are greater than $\sigma(2 i)$. Let $\min w_{i}^{\prime}$ (resp. min $w_{i}^{\prime \prime}$ ) denote the minimum letter of $w_{i}^{\prime}$ (resp. of $\left.w_{i}^{\prime \prime}\right)$. Then $\sigma$ is called an alternating André permutation if it is alternating and satifies $\min w_{i}^{\prime}>\min w_{i}^{\prime \prime}$ for every $i=1,2, \ldots, n$. The number of alternating André permutations of order $(2 n+1)$ is equal to $t_{n}$ (see, e.g., [FS71], Property 2.6 and (5.4), or [Po89]). The alternating André permutations of order 5 are the following: $53412,51423,41523,31524$. Let $A_{2 n+1, j}$ denote the set of alternating André permutations of order $(2 n+1)$ ending with $j$.

Thanks to the identity

$$
d_{n, j}(1)=\sum_{i \geq 0}\left(\begin{array}{c}
2 n+1-j \\
2 i+1
\end{array}\right) t_{i} \sum_{k=0}^{j-1} d_{n-i-1, k}(1),
$$

valid for $n \geq 1,2 \leq j \leq 2 n-1\left(d_{0, j}(1)=\delta_{0, j}\right)$, derived from the bivariable generating function (1.8), Christiane Poupard proved that

$$
\# A_{2 n+1, j}=d_{n, j}(1) \text {. }
$$


Three questions arise:

(1) Construct a natural (?) bijection of $\mathcal{N}_{2 n+1, j}^{0}$ onto $A_{2 n+1, j}$.

(2) Find an adequate statistic "stat" on the alternating André permutation such that holds the identity

$$
d_{n, j}(q)=\sum_{\sigma \in A_{2 n+1, j}} q^{\text {stat } \sigma} .
$$

(3) As mentioned in the Introduction, we have an expression for the generating function for $d_{n, j}(1)$ (formula (1.8)) and for $d_{n, j}(0)$ (formula $(1.14)$ ). Find the exponential (or factorial) generating function for $d_{n, j}(q)$.

\section{References}

[Cal05] Callan, David, A reference given in On-Line Encyclopedia of Integer Sequences for the sequence A009766.

[Ca54] Carlitz, Leonard, $q$-Bernoulli and Eulerian numbers, Trans. Amer. Math. Soc., 76 (1954), pp. 332-350.

[Ca75] Carlitz, Leonard, A combinatorial property of $q$-Eulerian numbers, Amer. Math. Monthly, 82 (1975), pp. 51-54.

[De04] Deutsch, Emeric, A reference given in On-Line Encyclopedia of Integer Sequences for the sequence A009766.

[FH00] Foata, Dominique; Han, Guo-Niu, Word straightening and $q$-Eulerian Calculus, Contemporary Mathematics, $q$-Series from a Contemporary Perspective, AMS, M. E. H. Ismail, D. W. Stanton Ed., 254, 2000, 141-156.

[FH08] Foata, Dominique; Han, Guo-Niu, Doubloons and new $q$-tangent numbers, preprint, 17 p., 2008.

[FS71] Foata, Dominique; Schützenberger, Marcel-Paul, Nombres d'Euler et permutations alternantes. Manuscript (unabridged version) 71 pages, University of Florida, Gainesville, 1971.

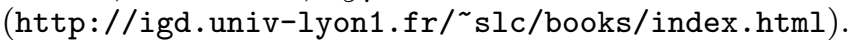

[FS71a] Foata, Dominique; Schützenberger, Marcel-Paul, Nombres d'Euler et permutations alternantes, in J. N. Srivastava et al. (eds.), A Survey of Combinatorial Theory, North-Holland, Amsterdam, 1973, pp. 173-187.

[FS73] Foata, Dominique; Strehl, Volker, Euler numbers and variations of permutations, in Colloquio Internazionale sulle Teorie Combinatorie, 1973, Tome I (Atti dei Convegni Lincei, 17, 119-131). Accademia Nazionale dei Lincei, 1976.

[FS78] Foata, Dominique; Schützenberger, Marcel-Paul, Major Index and Inversion number of Permutations, Math. Nachr., 83 (1978), pp. 143-159.

[Gz08] Graham, Ron; Zang, Nan, Enumerating split-pair arrangements, J. Combin. Theory, Ser. A, 115 (2008), pp. 293-303.

[Ha92] Han, Guo-Niu, Calcul Denertien, Thèse de Doctorat, Publ. I'I.R.M.A., Strasbourg, 476/TS-29, 1991, 119 pages http://www-irma.u-strasbg.fr/ guoniu/papers/p05these.pdf. 
[Ha92b] Han, Guo-Niu, Une courte démonstration d'un résultat sur la $Z$-statistique, $C$. R. Acad. Sci. Paris, 314, Sér. I (1992), pp. 969-971.

[Ha94] Han, Guo-Niu, Une transformation fondamentale sur les réarrangements de mots, Adv. in Math., 105(1) (1994), pp. 26-41.

[Po89] Poupard, Christiane, Deux propriétés des arbres binaires ordonnés stricts, Europ. J. Combin., 10 (1989), pp. 369-374.

[S107] Sloane, N. J. A., On-line Encyclopedia of Integer Sequences.

Dominique Foata

Institut Lothaire

1 , rue Murner

F-67000 Strasbourg, France

foata@math.u-strasbg.fr
Guo-Niu Han

I.R.M.A. UMR 7501

Université Louis Pasteur et CNRS

7 , rue René-Descartes

F-67084 Strasbourg, France

guoniu@math.u-strasbg.fr 